\title{
Anestésicos locais em clínica universitária odontológica: conhecimento dos graduandos acerca de efeitos adversos e tóxicos
}

\section{Local anesthetics in university dental clinic: knowledge of graduates about adverse and toxic effects}

Lorena de Queiroz Silva, John Elvys Siva da Silveira, Luiz Filipe Barbosa Martins, Edmir Geraldo de Siqueira Fraga, Sandna Larissa Freitas dos Santos, Hérick Hebert da Silva Alves, Karla Bruna Nogueira Torres Barros, Kaléu Mormino Otoni, Edilson Martins Rodrigues Neto

\section{RESUMO}

Como citar este artigo: SILVA, Lorena; SILVEIRA, John; MARTINS, Luiz; FRAGA, Edmir; SANTOS, Sandna; ALVES, Hérick; BARROS, Karla; OTONI, Kaléu; NETO, Edilson. Anestésicos locais em clínica universitária odontológica: conhecimento dos graduandos acerca de efeitos adversos e tóxicos. Revista Saúde (Sta. Maria). 2019; 45 (2).

\section{Autor correspondente:} Nome: Lorena de Queiroz Silva E-mail: lorenaqx@hotmail.com Telefone: (85) 996488173

Formação Profissional: Farmacêutica pelo Centro Universitário Católica de Quixadá (Unicatólica), Quixadá, CE, Brasil.

Filiação Institucional: Centro Universitário Católica de Quixadá

Endereço para correspondência: Avenida Tabelião Eneas de Lima

$n^{\circ}$ : 403C Bairro: Centro

Cidade: Quixadá Estado:

Ceará

CEP: 63.900-000

Data de Submissão:

02/05/2019

Data de aceite:

24/07/2019

Conflito de Interesse: Não há conflito de interesse

\section{(cc) $\mathrm{BY}-\mathrm{NC}-\mathrm{ND}$}

Este trabalho objetivou verificar o conhecimento dos graduandos do curso de odontologia sobre a utilização correta e segura de anestésicos locais na clínica odontológica. Inicialmente os acadêmicos do $9^{\circ}$ e $10^{\circ}$ semestre responderam a um questionário com finalidade de observar suas indicações para os pacientes, de acordo com sua escolha anestésica e mensurar seus conhecimentos sobre possíveis reações adversas e tóxicas relacionadas a estes compostos. Após a verificação das respostas, estas foram classificadas de maneira semiqualitativa. Os dados coletados foram inseridos no banco de dados do Excel, para viabilizar o processo das respostas obtidas. Constatou-se maior número de acertos, nas questões relativas à pacientes gestantes, e maior quantidade de erros observados foi predominantemente em questões sobre anafilaxia em geral e diabéticos insulinodependentes. De acordo com a observação das respostas obtidas, conclui-se que os acadêmicos demonstraram dificuldades sobre o tema proposto, principalmente quando se trata de casos com pacientes com algum tipo de deficiência, como por exemplo, alérgicos. Assim, se faz necessário além do conhecimento da substância correta, que o cirurgião dentista observe a história clínica do paciente para prosseguir 0 atendimento, buscando evitar quando possível padronização. A técnica e a quantidade correta de solução, tendo em vista que se evite administração intravascular, por exemplo, que pode ser evitada com a aspiração prévia, visando também à necessidade do seguimento de instruções protocoladas.

PALAVRAS-CHAVE: Anestesia. Complicações. Odontologia. Toxicologia.

\section{ABSTRACT}

This study aimed to verify the knowledge of undergraduate dentistry students about the correct and safe use of local anesthetics in the dental clinic. Initially the students of the 9th and 10th semester answered a form to observe their indications for the patients, according to their anesthetic choice and to measure their knowledge about possible adverse and toxic reactions related to these compounds. After verifying the answers, they were classified in a semi-qualitative manner. The collected data were entered in the Excel database, to enable the process of the obtained answers. There was a greater number of correct answers in the questions related to pregnant patients, and a larger number of errors observed was predominantly in questions about anaphylaxis in general and insulin dependent diabetics. According to the observation of the answers obtained, it is concluded that the students demonstrated difficulties on the proposed theme, especially when dealing with cases with patients with some type of disability, such as allergic patients. Thus, it is necessary beyond the knowledge of the correct substance, that the dentist observe the patient's clinical history to continue the care, seeking to avoid when possible standardization. The technique and the correct amount of solution, in order to avoid intravascular administration, for example, which can be avoided with prior aspiration, also aiming at the need to follow protocol instructions. 


\section{INTRODUÇÃO}

Os Anestésicos Locais (ALs) são substâncias químicas que agem através do bloqueio dos canais de sódio (Na+), inativando-os, ocasionando redução da taxa de potencial de ação e sua velocidade de despolarização resultando em falha de condução e bloqueio nervoso, sendo os fármacos mais utilizados em odontologia (MALAMED, 2013). Agentes Vasocontritores (VCs) são adicionados aos ALs para prolongar a duração da anestesia e controle da hemostasia, levando à minimização do risco de toxicidade sistêmica e hemorragias, respectivamente. Apesar das vantagens dos VCs, a literatura alerta para alguns possíveis efeitos colaterais (MALAMED, 2013).

Acredita-se que por existirem no mercado uma gama de soluções e concentrações diferentes disponíveis, muitos cirurgiões dentistas optam por padronizar apenas um tipo de solução para todos os procedimentos, o que pode trazer malefícios dependendo do estado de saúde dos pacientes submetidos a tais (GAUJAC, 2017).

$\mathrm{Na}$ Dinamarca, em uma clínica especializada em alergologia, observou-se que dentre todas as reações notificadas, apenas $2,5-10 \%$ destas são relacionadas a alergias imediatas a ALs, sendo consideradas raras entre especialistas da área. Outros estudos sugerem que a incidência de alergia imediata varia apenas entre 0-1\% (KVISSELGAARD et al., 2017; YILMAZ et al., 2018). Há indícios de que a toxicidade por estes compostos esteja relacionada com administração inadequada, causando efeitos indesejáveis para o paciente, todavia, deve-se considerar que a maioria destes eventos permanecem negligenciados (OLIVEIRA, SIMONE; RIBEIRO, 2010).

Alérgicas, cardiotoxicidade, neurotoxicidade, hematotoxicidade são consideradas as reações sistêmicas mais recorrentes. Contudo, apesar do levantamento sobre os registros de reações adversas a medicamentos relacionados a ALs, relatou que ainda não se avaliou reportes à entidade reguladora brasileira, sugerindo que não há estudos com essa perspectiva a nível de Brasil, onde é possível encontrar apenas dados epidemiológicos regionais. Em estudos recentes com acadêmicos e cirurgiões dentistas, foram observados que os mesmos obtiveram conhecimento limitado sobre essas substâncias (RODRIGUES NETO et al., 2014; PINHEIRO et al., 2015; PONTANEGRA et al., 2017).

Diante do exposto, este trabalho objetivou mensurar o conhecimento dos graduandos do curso de odontologia sobre a utilização correta e segura de anestésicos locais na clínica universitária odontológica.

\section{MÉTODO}

Tratou-se de um estudo descritivo, exploratório, transversal com abordagem qualiquantitativo, onde os dados de interesse foram obtidos por meio de um questionário com questões abertas e em múltipla totalizando 8 perguntas, Após recebimento do questionário devidamente preenchido, foram avaliadas as respostas dos mesmos sobre a utilização dos 
anestésicos em diferentes grupos e seu reconhecimento quanto a reações adversas e tóxicas, aplicadas aos acadêmicos de odontologia de uma Universidade localizada na cidade de Quixadá, localizada no Sertão Central do Ceará.

Participaram da pesquisa os do $9^{\circ}$ e $10^{\circ}$ semestre, totalizando 38 participantes. Adotaram-se como critérios de inclusão, os acadêmicos do curso de odontologia de ambos os sexos que estivessem devidamente matriculados na universidade de realização do estudo, presentes no momento da coleta e que já cursaram a disciplina de farmacologia.

Os dados foram inseridos no banco de dados do Software Microsoft Exceß versão 16.0.6769.2017 para viabilizar o processamento e análise das respostas obtidas, onde a tabulação foi feita pelo método de Statistical Package for the Social Sciences (SPSS) versão 24.0 para Windows e a significância estatística dos dados foi estipulada em $5 \%(p<0,05)$.

A pesquisa foi submetida ao Comitê de Ética em Pesquisa do Centro Universitário Católica de Quixadá, através da Plataforma Brasil sendo avaliado de acordo com a Resolução 466/12 do Conselho Nacional de Saúde, que regulamentam Diretrizes e Normas sobre pesquisa envolvendo seres humanos (BRASIL, 2012), e aprovados com parecer de número 2.853.203.

\section{RESULTADOS E DISCUSSÕES}

A instituição conta com 205 acadêmicos devidamente matriculados no curso de odontologia, onde de acordo com os critérios de inclusão já citados apenas 38 (18,5\%) participaram da pesquisa, sendo $14(37 \%)$ do $9^{\circ}$ semestre, e 24 do $10^{\circ}$ semestre $(63 \%)$.

Com relação a primeira das oito questões elaboradas, era preciso citar uma alternativa anestésica para pacientes que são alérgicos a Benzocaína, onde 39\% das respostas estavam corretas e $41 \%$ incorreta enquanto $20 \%$ não sabem, ou não responderam. Dos acertos, foi observado que os participantes indicaram outros anestésicos injetáveis do grupo amida, e os erros são justificados já que se esperava, que neste caso, os acadêmicos optassem pela mesma forma farmacêutica. Atualmente as amidas são mais utilizadas na prática clínica por apresentarem mais segurança que os Ésteres, pois o metabolismo da maioria destes compostos resulta na produção de ácido para-aminobenzóico (PABA), associado à maioria das reações alérgicas (CARVALHO et al., 2013; MALAMED, 2013).

A segunda questão pedia para que o participante citasse uma alternativa para pacientes alérgicos à bissulfito de sódio (antioxidante do vasoconstritor) que necessitam passar por procedimento cirúrgico mais prolongado. 0 bissulfito de sódio é uma substância componente de alguns tubetes com Vasoconstritores (VCs), apresentando finalidade antioxidante, geralmente é adicionada em soluções que contenham VCs adrenérgicos, como epinefrina, norepinefrina, corbadrina e fenilefrina (ANDRADE, 2014). Com relação a este composto, na segunda questão foi observado que $42 \%$ responderam corretamente, desses, $36 \%$ responderam mepivacaína e $6 \%$ prilocaína, quanto $47 \%$ não acertaram e $11 \%$ não sabem, ou não responderam. 
Atenta-se que para tal pergunta $5 \%$ de participantes optaram por Lidocaína com Epinefrina, onde nesse contexto seria uma opção inviável, pois como expõe Malamed (2014), o bissulfito de sódio componente presente em tubetes com epinefrina tem relacionando-se com reações anafiláticas principalmente em diabéticos glicocorticoidedependentes (urticária, angioedema e exacerbação da asma). Segundo o mesmo autor, uma opção para tal seria administrar anestésicos que não contenham VCs, como prilocaína 4\% oumepivacaína 3\%, ambos em forma de cloridrato, considerando as ações prolongadas das mesmas. Já como observado na obra de Andrade (2014), além das opções anteriores, poderia ser utilizado para tal, prilocaína $3 \%$ com felipressina 0,03 Ul/mL, considerando que este VC não é pertencente do grupo das aminas, não apresentando nenhum sulfito em sua formulação.

O anestésico de escolha para pacientes com Hipertensão Arterial descompensada foi assunto da terceira questão onde observou-se $40 \%$ de acertos, $2 \%$ não souberam responder e $58 \%$ dos participantes erraram ao optar por utilizar anestésicos contendo VCs da classe das aminas.

Apesar de serem anestésicos de ação local e espere-se ação vasoconstritora apenas no local de aplicação, uma parte da substância atinge ação sistêmica levando a diversos sintomas como aumento de PA. A epinefrina, por exemplo, promove constrição através da estimulação de receptores a1 podendo interagir também com receptores $\beta 1$ e $\beta 2$, aumentando a frequência cardíaca, força de contração e consumo de oxigênio pelo miocárdio, e dilatação dos vasos sanguíneos da musculatura esquelética, respectivamente Dessa maneira, em pacientes que contenham hipertensão não controlada, o uso de VCs deve ser evitado quando possível (MURINELLO, 2001; CATTERALL; MACKIE, 2012).

$\mathrm{Na}$ questão posterior, foi questionado qual o anestésico de escolha para o tratamento em gestantes, obteve-se $80 \%$ de respostas corretas e $20 \%$ de erros. Com base no estudo apresentado por Vasconcelos et al. (2012), geralmente a maioria dos anestésicos possuem plena segurança, podendo ser utilizados em qualquer período da gestação, já que em sua maioria, apresentam apenas Risco B (de acordo com a classificação do FDA), com exceção da mepivacaína, articaína, benzocaína (tópico) e bupivacaína que apresentam classificação de Risco C.

Contrariamente, outros autores alertam que atendimentos odontológicos devem ser evitados até o terceiro trimestre de gravidez, porém quando urgentes a substância mais indicada e empregada na rotina odontológica para tal é Lidocaína 2\% com epinefrina 1:100.000, visando que a utilização do VC diminuiria a necessidade de reaplicação das substâncias, apresentando-se como uma substância com baixa toxicidade sistêmica e mínimo potencial alérgico (CANEPPELE et al., 2011.; VASCONCELOS et al., 2012; CARVALHO, 2014; RODRIGUES NETO et al., 2014).

Quando questionados a respeito dos anestésicos mais relacionados com eventos anafiláticos, sobre essa quinta questão o resultado foi apenas $3 \%$ acertaram e um total alarmante de $92 \%$ de erros, além de $4 \%$ que não responderam. Sabe-se que a inserção dos anestésicos do grupo amida e a diminuição da utilização dos ésteres propiciaram com que 
menos eventos anafiláticos viessem a ocorrer, uma vez que a metabolização das aminas não produz o PABA (ácido para-aminobenzóico), substância associada à maioria dos eventos anafiláticos. Assim, quando detectadas tornam todos os anestésicos deste grupo inviáveis para uso deste paciente, vista a reação alérgica cruzada; fenômeno que não ocorre com as amidas, podendo assim ser utilizado outro anestésico desta classe (MOORE; HERSH 2010; ANDRADE, 2014; RODRIGUES NETO et al., 2014).

Quando indagados na sexta questão sobre suas indicações para pacientes diabéticos insulino dependentes, 11\% acertaram, 85\% erraram e 4\% não responderam. Fabris et al. (2018) relatam que a utilização de anestésicos com VC são benéficos, visando que o mesmo aumentaria o tempo do efeito anestésico, onde a opção mais utilizada é a epinefrina 1:100.000, porém há controvérsias. Em estudos realizados com pacientes diabéticos pré e pós-cirúrgicos, foi observado que uso de epinefrina altera os níveis mínimos de glicose sanguínea, principalmente em pacientes usuários de medicamentos hipoglicemiantes e insulina que camuflam esta reação, sendo anestésicos sem VC as escolhas mais seguras (ARONOVICH et al., 2010). Para Fabris (2018), a felipressina mostra-se uma opção segura já que não altera a glicemia.

Os anestésicos que apresenta relação com o aparecimento de metahemoglobinemia, foi o problema tratado na sétima pergunta e com relação a esse tema $63 \%$ acertaram, $12 \%$ erraram e $25 \%$ não responderam. A MetHb é uma condição clínica rara, podendo ser hereditária ou adquirida, sendo a cianose e redução da saturação periférica do oxigênio, os aspectos mais marcantes. Alguns fatores de risco incluem idade jovem, mutações genéticas como a baixa atividade do citocromo B5 redutase, deficiência de glicose-6-fosfatase (G6P) e também pela administração de medicamentos, como oxido nítrico, sulfonamidas, dapsona, metroclopramida, nitrofurantoína e anestésicos locais (MURINELLO, 2001; EROL et al., 2018).

Nas evidências apresentadas em três relatos feitos por Erolet al. (2018), três bebês apresentaram cianose após poucas horas de nascimento, nos quais após investigação e exclusão de outras possíveis causas, concluiu-se que 0 causador do fato se tratava do anestésico que havia sido administrado nas gestantes (Prilocaína). O ocorrido foi resolvido após tratamento com Ácido Ascórbico. Como relata Rodrigues et al. (2017), este problema associa-se frequentemente aos anestésicos prilocaína, articaína, benzocaína (uso tópico) e lidocaína.

As reações adversas foi assunto da oitava e última questão. A escolha desse a assunto se justifica por estarem geralmente relacionadas com a administração excessiva de determinado medicamento, no caso dos anestésicos locais, esses efeitos podem trazer várias complicações para o paciente. Diante disso foi indagado aos entrevistados sobre as reações mais comuns que estão relacionadas aos anestésicos locais, ambos descrito no Gráfico 01 abaixo. 
Gráfico 01: Principais reações adversas dos anestésicos locais relatadas pelos estudantes do $9^{\circ}$ e $10^{\circ}$ do curso de Odontologia, Unicatólica, 2018.
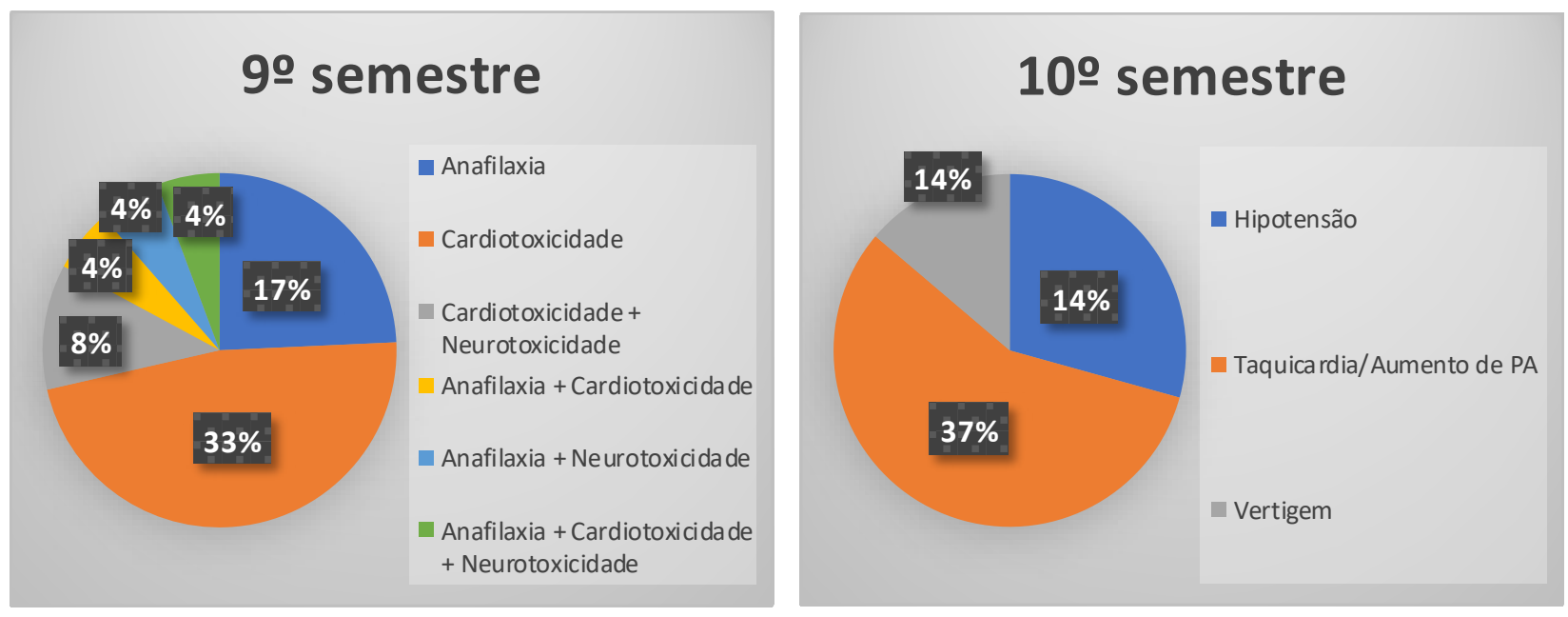

Quando um fármaco é administrado, podem ser observados efeitos desejados tão quanto os não desejados, mesmo que mínimos, já que nenhumo fármaco é totalmente específico (BRASIL, 2015). Em uma revisão sistemática realizada por LIU etal. em 2013, observou-se que a maioria dos casos de intoxicação por anestésicos estavam relacionados com pessoas que continham doenças crônicas como: doenças miocárdicas, desequilíbrio eletrolítico, acidose, diabetes, doenças da tireoide, anemia.

Quando questionados sobre as reações adversas mais relacionadas com anestésicos, a grande maioria respondeu anafilaxia (24\%), e Cardiotoxicidade (23\%), enquanto $30 \%$ não souberam ou não responderam. Gomez e Araque (2011) classificam as reações tóxicas em: localizadas ou sistêmicas (alérgicas); sistêmicas (cardiovasculares, SNC, metahemoglobinemia); localizadas (toxicidade tecidual). Já na classificação utilizada por Rodrigues Neto et al. (2014) foram observadas de forma mais específica: alérgicas, cardiotoxicidade, neurotoxicidade; hematotoxicidade e mistas.

Os efeitos cardiotóxicos estão relacionados com o aparecimento de arritmias e depressão miocárdica de difícil reversão principalmente quando a bupivacaína for o agente utilizado, além de bradicardias e taquiarritmias ou fibrilação. Como possuem características capazes de atravessar com facilidade a barreira hematoencefálica, os ALs geralmente podem levar à ação depressora no sistema nervoso. Em doses tóxicas ou em sobredosagem a manifestação clínica primária é a convulsão tônicoclônica generalizada, porém são incluídos também sinais e sintomas como entorpecimento da língua, sonolência e tonturas (OLIVEIRA; SIMONE; RIBEIRO, 2010; UDELSMANN et al., 2012; PINHEIRO et al., 2015). 
$\mathrm{Na}$ hematotoxicidade, a reação mais comum que se apresenta é a metahemoglobinemia, que apesar de rara pode acometer principalmente as gestantes. Esta ocorre pela decorrência da oxidação do $\mathrm{Fe}^{+2}$ (ferro ferroso) em $\mathrm{Fe}^{+3}$ (ferro férrico) na molécula de Hemoglobina $(\mathrm{Hb})$, tornando-a incapaz de captar oxigênio e transportar o oxigênio, levando a consequente cianose pelo acúmulo de metahemoglobina no sangue. Sintomas como dispneia, náusea e cefaleia geralmente também aparecem (MURINELLO, 2001).

Nas reações mistas, acontecem pela capacidade que o anestésico tem de atuar em vários sítios de ação, onde além de sua ação nos neurônios também podem realizar bloqueio neuromuscular. Onde, apesar de pouco relevante, em algumas situações este pode juntamente com relaxantes musculares (despolarizantes e não-despolarizantes) levar a períodos prolongados de paralisia muscular. Da mesma maneira, juntamente com medicamentos depressores do SNC, levar à potencialização de seus efeitos depressores (RODRIGUES NETO et al., 2014).

Os efeitos localizados foram observados principalmente pela toxicidade tissular, que na maioria das vezes são causadas por traumas e hemorragias decorrentes da administração dessas substâncias. Em um estudo foi relatado que este risco se tem relacionado principalmente com anestésicos como bupivacaína, procaína, tetracaína e ropivacaína, por ordem detoxicidade relacionada (TASCH; BUTTERWORTH, 2006; GOMEZ; ARAQUE, 2011).

\section{CONSIDERAÇÕES FINAIS}

Diante do exposto, percebe-se que há uma deficiência no entendimento por parte dos acadêmicos sobre o tema proposto, principalmente no que norteia casos relacionados com pacientes especiais. Muitas vezes os profissionais tendem a padronizar o anestésico a ser utilizado na maioria dos procedimentos, contudo, o conhecimento sobre outras possibilidades se faz necessária principalmente quando se observa que os anestésicos são substâncias que podem levar a uma gama de efeitos adversos graves. Tendo em vista que são as substâncias mais utilizadas pelos dentistas, é notável a importância de que estes devem receber atenção, e devem ser escolhidos de acordo com o estado de saúde dos pacientes e suas contraindicações, levando ainda em consideração a particularidade de cada caso.

Para os acadêmicos é notória a necessidade de que se intensifique o estudo com relação a estes compostos, pois se observou que é essencial além do conhecimento da substância correta, que o cirurgião dentista observe a história clínica do paciente, para prosseguir o atendimento utilizando a técnica e a quantidade correta e necessária de solução, tendo em vista ainda que se evite administração intravascular, por exemplo, podendo usar da aspiração prévia, e ainda a necessidade do seguimento de instruções protocoladas. 


\section{REFERÊNCIAS}

1. ANDRADE, E. D. Terapêutica medicamentosa em odontologia. 3. ed. São Paulo: Artes Médicas, 2014.

2. ARONOVICH, B. et al. The relationship of Glycemic Control of the Outcomes of Dental Extractions. Journal Oral Maxillofacial Surg, v.12, n.68, p.2955-2961, dez. 2010.

3. CANEPPELE, T. M. F. et al. Conhecimento dos cirurgiões-dentistas sobre 0 atendimento de pacientes especiais: hipertenso, diabéticos e gestantes. Journal of Biodentistry and Biomaterials, São Paulo, n. 1, p. 31-41, mar.l ago. 2011.

4. CARVALHO, B. et al. O emprego dos anestésicos locais em odontologia: revisão de literatura. Revista Brasileira de Odontologia, Rio de Janeiro, v. 70, n. 2, p. 178-181, jul./dez. 2013.

5. CATTERALL, W.; MACKIE, K. Anestésicos locais. In: As bases farmacológicas da terapêutica de Goodman \& Gilman. 12. ed. Porto Alegre: McGraw-Hill, 2012. p. 279-292.

6. EROL, S. et al. Transient Methemoglobinemia in three Neonates due to Maternal Pudendal Anesthesia. Revista Brasileira de Odontologia, v. 27, n. 12, p. 783-784, 2017.

7. FABRIS, C. et al. Conhecimento dos cirurgiões dentistas sobre o uso de anestésicos locais em pacientes: diabéticos, hipertensos, cardiopatas, gestantes e com hipertireoidismo Journal of Oral Investigations, Passo Fundo, v. 7, n. 1, p. 33-51, jan./jun. 2018.

8. GAUJAC, $\mathrm{C}$ et al. Reações alérgicas medicamentosas no consultório odontológico. Revista de Odontologia da Universidade Cidade de São Paulo, v. 21, n. 3, p. 268-276, 2017.

9. KVISSELGAARD, A. D. et al. Risk of Immediate-Type Allergy to Local Anesthetics Is OverestimatedResults from 5 Years of Provocation Testing in a Danish Allergy Clinic. Journal of Allergy and Clinical Immunology: In Practice, v. 6, n. 4, p. 1217-1223, 2018. 
10. LIU, W. et al. Adverse drug reactions to local anesthetics: a systematic review. Oral and maxillo facial surgery, Chengdu: Elservier, v. 115, n. 3, p. 319-327, 2013.

11. MALAMED, S. F. Manual de anestesia local. 6. ed. São Paulo: Elsevier, 2013.

12. MOORE, P.A.; HERSH, E.V. Local Anesthetics: Pharmacology and Toxicity. Dental Clinical North Americain, v. 4,n.54, p. 587-599, oct. 2010.

13. MURI, E.M.F.; SPOSITO, M.M.M.; METSAVAHT, L. Efeitos secundários potencialmente desejáveis dos anestésicos locais. Acta Fisiatrica,v.1, n. 17, p. 28-33, 2010.

14. MURINELLO, A. et al. Meta-hemoglobinemia. Med Int, v. 8, n. 4, p. 210. 2001.

15. OLIVEIRA, A.E.M.; SIMONE, J.L.; RIBEIRO, R.A. Pacientes hipertensos e a anestesia na odontologia: devemos utilizar anestésicos locais associados ou não com vasoconstritores? HU Revista, Juiz de fora, v. 36, n. 1, p. 69-75,jan./mar. 2010.

16. PINHEIRO, A.C.et al. Dentists' knowledge regarding signs and symptoms of the systemic toxicity of local anesthetic solutions.Revista Gaúcha de Odontologia, Porto Alegre, v.63, n. 1, p. 41-46, jan./mar. 2015.

17. PONTANEGRA, R. S. M, et al. Análise do conhecimento de Graduandos em Odontologia sobre o uso de anestésico local em pacientes com necessidades especiais. FOL - Faculdade de Odontologia de Lins/Unimep, v. 27, n. 1, p. 5-14, jan.jjun. 2017.

18. RODRIGUES, F. et al. Anestesia local em gestantes na odontologia contemporânea. Journal Health NPEPS, v. 2, n. 1, p. 254-271, 2017.

19. RODRIGUES NETO, E. M. et al. Toxicidade de anestésicos locais na prática clínica. Revista Eletrônica de Farmácia, Ceará, v. 11, n. 1, p. 47-59, fev. 2014.

20. TASCH, M.D.; BUTTERWORTH, J.F. Toxicity of local anesthetics. [S.I.]: Asa Refreshing Course, 2006. 
21. UDELSMANN, A. et al. Lipids in local anesthetic toxicity. ABCD, Arquivo Brasileiro de Cirurgia Digestiva, v. 25, n.3, p.169-172, 2012.

22. YILMAZ, I. et al. Local anesthetics allergy: who should be tested? European Annals of Allergy and Clinical Immunology, v. 50, n. 02, p. 66-71, 2018. 